\title{
Prognostic value of shape-descriptive factors for the progression of geographic atrophy secondary to age- related macular degeneration
}

Maximilian Pfau ${ }^{1 *}$, Moritz Lindner ${ }^{1,2 *}$, Lukas Goerdt ${ }^{1}$, Sarah Thiele ${ }^{1}$, Jennifer Nadaß ${ }^{\beta}$, Matthias

Schmid ${ }^{3}$, Steffen Schmitz-Valckenberg ${ }^{1}$, SriniVas R. Sadda ${ }^{4}$, Frank G. Holz ${ }^{1}$, Monika Fleckenstein ${ }^{1}$

for the Fundus Autofluorescence in Age-Related Macular Degeneration Study Group

1. Department of Ophthalmology, University of Bonn, Germany

2. The Nuffield Laboratory of Ophthalmology, Sleep and Circadian Neuroscience Institute, Nuffield Department of Clinical Neurosciences, University of Oxford, Oxford, United Kingdom

3. Institute for Medical Biometry, Informatics and Epidemiology, University of Bonn, Germany

4. Doheny Eye Institute, University of California Los Angeles (UCLA), USA

* These authors contributed equally to this work.

Running title: Lesion configuration and GA progression

Number of words: $\quad 4,007$

Number of figures: $\quad 5$

Number of tables: $\quad 3$

Supplemental Digital Content: $\quad 4$

Correspondence:

PD. Dr. Monika Fleckenstein

Department of Ophthalmology

University of Bonn

Ernst-Abbe-Str. 2

53127 Bonn

Germany

Tel.: $\quad$ +49228 28716826

Fax: $\quad$ +49 22828711470

E-mail: monika.fleckenstein@ukbonn.de

\section{Funding:}

The authors have no proprietary interest. Heidelberg Engineering (Heidelberg, Germany) provided research material (Heidelberg Spectralis HRA + OCT). Heidelberg Engineering had no role in the design or conduct of this study.

BONFOR GEROK Program, Faculty of Medicine, University of Bonn, Grant No O-137.0022, O-137.0025 to MP and Grant No O-137.0020 to ML

DFG Grant FL 658/4-1 and FL 658/4-2

DFG Grant Ho1926/3-1

The sponsor or funding organizations had no role in the design or conduct of this research.

\section{Acknowledgements:}

The authors thank Suzan E. Hunt for language editing and proofreading. 


\section{Key words}

age-related macular degeneration, circularity, fundus autofluorescence, geographic atrophy, perimeter, progression rates, perimeter

\section{Summary statement}

Fundus-autofluorescence imaging allows for fully automated prediction of geographic atrophy progression rates based on annotations that are already used in clinical routine and that are part of the eligibility assessment of currently ongoing trials. 


\section{Abstract \\ Purpose:}

To systematically compare the prognostic value of multiple shape-descriptive factors in the natural course of the disease.

\section{Methods:}

A total of 296 eyes of 201 patients (female 130; mean age: $72.2 \pm 13.08$ years) with a median follow-up of 2.38 years from two prospective, non-interventional natural history studies (Fundus-Autofluorescence-in-Age-related-Macular-Degeneration [clinicaltrials.gov identifier NCT00393692], Directional-Spread-in-Geographic-Atrophy [NCT00393692]) were included in the analysis. Serial fundus-autofluorescence (FAF) images were annotated using semiautomated image analysis software to determine the lesion area, circularity, perimeter and caliper diameters. These variables and the FAF phenotype were evaluated for prediction of the future square-root progression-rates using linear mixed-effects models.

\section{Results:}

For the combined model, leave-one-out cross validation (LOO-CV) on patient level (scenario1: previously unknown patient) resulted in a goodness-to-fit ( $R^{2}$-value) of 0.244 and LOO-CV on visit level (scenario2: prior observation of the patient) in a $R^{2}$-value of 0.391 . This indicated that shape-descriptive factors could explain $24.4 \%$ of the variance in GA progression in previously unknown patients and $39.1 \%$ in patients with prior observation.

\section{Conclusions:}

These findings confirm the relevance of shape-descriptive factors and previous progression as prognostic variables for GA progression. However, a substantial part of the remaining variation in GA progression appears to depend on other variables, some of which are visible in optical coherence tomography. 


\section{Introduction}

Age-related macular degeneration (AMD) is the leading cause of blindness in developed countries. ${ }^{1,2}$ Choroidal neovascularization (CNV) and retinal angiomatous proliferation (type 3 neovascularization) in its exudative late stage manifestation are the most common cause of severe visual loss in AMD. ${ }^{1,3,4}$ However, at least $20 \%$ of AMD patients who are legally blind have lost central vision due to geographic atrophy (GA), the non-exudative late stage manifestation of AMD. ${ }^{5-10}$

The term GA in the context of AMD (or "senile macular choroidal degeneration") was originally introduced by Gass in 1970 to describe the funduscopic appearance of circumscribed areas of outer retinal atrophy..$^{5,11,12}$ Histologically, GA is characterized by loss of retinal pigment epithelium (RPE) as well as a degeneration of the outer layers of the neurosensory retina and the choriocapillaris. ${ }^{13,14}$ Over time, foci of GA tend to slowly enlarge and coalesce with an eventual involvement of the fovea. 5,11 Among other pathogenetic mechanisms, chronic inflammatory processes, excessive lipofuscin accumulation, complement system dysregulation, and vascular factors have been implicated in the development of AMD. ${ }^{9,14,15}$

On fundus autofluorescence (FAF) imaging, areas of GA are clearly defined due to RPE degeneration and the absence of therein contained fluorophores allowing for semi-automated detection and area quantification of atrophic lesions. ${ }^{16-21}$ Change in GA area over time as assessed by FAF imaging has been accepted as a clinical endpoint by the U.S. Food and Drug Administration and served as primary outcome measure in the so far largest conducted trials Chroma (ClinicalTrials.gov Identifier: NCT02247479) and Spectri (NCT02247531). ${ }^{22}$

By using FAF imaging, stereo color fundus photographs (CFP) or spectral domain optical coherence tomography (SD-OCT), various lesion- and eye-specific morphological characteristics have been shown to be associated with GA progression including lesion size,${ }^{23-31}$ previous lesion progression, ${ }^{32}$ lesion shape,$^{23,26,33-38}$ focality index, ${ }^{26,31,33-38}$ and lesion location. ${ }^{13,21,23,26,32,39}$ On the basis of findings from the FAM-study (Fundus 
Autofluorescence Imaging in Age-Related Macular Degeneration), different FAF subphenotypes could be identified that were also associated with differential progression rates. ${ }^{18,25,40}$ A similar classification of GA phenotypes consisting of three phenotypes has recently been suggested based on a data-driven cluster analysis approach. ${ }^{41}$ However, to date it is not known which of these factors have the strongest prognostic impact on GA progression. It has not yet been systematically investigated whether these factors are correlated to or represent the same underlying morphological alterations.

In this study a refined analysis of shape-descriptive factors including lesion area, perimeter, circularity, as well as caliper diameters and their impact on progression was performed based on semi-automatic FAF image analysis. We hypothesize that a large lesion circumference, a low circularity and a large lesion diameter are prognostic for rapid progression of GA secondary to AMD. 


\section{Methods}

\section{Patients}

Patients were recruited in the Fundus Autofluorescence Imaging in Age-Related Macular Degeneration (FAM) study (clinicaltrials.gov identifier, NCT00393692) and in its extension trial the Directional Spread in Geographic Atrophy (DSGA) study (NCT02051998). These non-interventional, prospective natural history studies adhered to the tenets of the Declaration of Helsinki and were approved by the institutional review boards of the participating centers. Written informed consent was obtained from each participant after explanation of the study's nature and possible consequences of participation. The current analysis included eyes from patients with unilateral or bilateral GA secondary to AMD and a history of serial examinations $\geq 6$ months. If both eyes met the inclusion criteria, both were included in the analysis.

\section{Definition of Geographic Atrophy Resulting from Age-Related Macular Degeneration}

In a first step, geographic atrophy was defined funduscopically as one or more well-defined, usually more or less circular patches of partial or complete depigmentation of the RPE, typically with exposure of the underlying large choroidal blood vessels. Further, in the FAM and DSGA-studies, GA resulting from AMD was defined as a sharply demarcated lesion $\left(\geq 0.05 \mathrm{~mm}^{2}\right)$ with clearly reduced FAF that did not correspond to exudative retinal changes (e.g., hemorrhage, exudates, fibrous scar) in an eye with funduscopically visible pigmentary abnormalities in conjunction with soft or reticular drusen. ${ }^{42}$ Eyes exhibiting the GPS ('fine granular with peripheral punctate spots') FAF pattern - associated with mutations in the $A B C A 4$ gene - or any other phenotypic features of known monogenic diseases (e.g. central areolar choroidal dystroph [PRPH2]) were excluded from the analysis. ${ }^{43,44}$ 


\section{Image Acquisition, Processing, and Grading}

FAF and NIR reflectance images were acquired using a HRA 2 or Spectralis device (Heidelberg Engineering, Heidelberg, Germany) as previously described. ${ }^{20}$ Delineation of GA areas was performed using the RegionFinder ${ }^{\mathrm{TM}}$ software (Heidelberg Engineering). ${ }^{20}$ The applied version (2.5.5.0) included a feature that automatically registers NIR reflectance images to the FAF images to facilitate the assessment of the foveal involvement. ${ }^{19-21}$ To annotate GA with the RegionFinder ${ }^{\mathrm{TM}}$ software, the readers selected the pixel with the lowest FAF signal (darkest gray value) of each GA lesion and increased the growth power to include adjacent pixels until the annotation just exceeded the lesion boundaries. Finally, the growth power was decreased by one increment below this threshold. ${ }^{20,45}$ Automated "vessel detection", "shadow correction" or manually placed constraints could be used to exclude retinal vessels or macula pigment from the GA annotation. ${ }^{20,45}$

The graded annotated images were transferred to ImageJ (Bethesda, Maryland, USA [Available at: https://imagej.nih.gov/ij/]) to measure the area, circularity and perimeter of the lesions with a custom plug-in. ${ }^{46}$ The plug-in looped through all annotation files, separated the annotations from manual constraints using the "Threshold Color" function, ran "Analyze Particles" to obtain the above mentioned measurements and saved these to a CSVformatted file. Scaling-factors for the pixel-to- $\mu \mathrm{m}^{2}$ conversion were obtained from the HEYEX software (Heidelberg Engineering). A subset of 100 randomly selected visits was graded by both readers to estimate the inter-rater reliability.

\section{Definition of the evaluated shape-descriptive factors}

The lesion area describes the total lesion including all foci of $\mathrm{GA}>0.05 \mathrm{~mm}^{2}$. The square root lesion area is defined as the square root of the lesion area. The (cumulative) perimeter describes the length of the cumulative circumference (i.e. perimeter) of all foci (Figure 1). The (cumulative) circularity was defined $a s^{36}$ : 


$$
\text { Circularity }=\frac{4 \times \pi \times \text { Area }^{2}}{\text { Perimeter }^{2}}
$$

The Feret $_{\max }$ diameter (maximum caliper diameter) was defined as the maximum perpendicular distance between parallel tangents touching opposite sides of the lesion. The Feret $_{\min }$ diameter (minimum caliper diameter) was defined as the minimum perpendicular distance between parallel tangents touching opposite sides of the lesion. ${ }^{47}$ The focality index described the number of foci with an area $>0.05 \mathrm{~mm}^{2}$. Furthermore, the $\boldsymbol{F A F}$ phenotypes were classified as previously specified. ${ }^{25}$ Briefly, the classification is based on FAF features in the junctional zone of atrophy in patients with GA. As a first step, the overall presence and topography of increased FAF in relation to the lesion boundary was rated as none, focal, banded, patchy or diffuse. ${ }^{25}$ Eyes with diffusely increased FAF could then be further sub-classified according to the pattern of increased FAF (reticular/branching, finegranular or trickling). ${ }^{25}$

\section{Statistical Analysis}

Data were compiled in a spreadsheet application and analyzed using the software environment $\mathrm{R}$, version 3.1.3. ${ }^{48}$ Inter-rater reliability between the two readers was assessed using interclass correlation coefficients (ICC) and the coefficient of repeatability $(1.96 \times \sqrt{2} \times$ Standard Error of Measurement) as recommended by Bland and Altman. ${ }^{49}$ Normally distributed continuous variables were presented as mean \pm standard deviation. Non-normal variables were described using the median and the 25\% (Q1) and 75\% (Q3) quartiles. Categorical variables were analyzed using frequency tables. Differences in age were tested for statistical significance using an unpaired t-test. The gender distribution among patients was compared using a chi-squared test. As suggested by Yehoshua et al., area measurements were square-root transformed to reduce the dependence of growth rates on the baseline size of GA. ${ }^{29,38}$ 
To quantify the effect of the shape-descriptive factors on lesion progression within the patient collective, linear mixed-effects models were used as described previously. The 2-level random effects models contained separate eye-specific and patient-specific effects and accounted for dependencies between measurements that were obtained from the same patient, eye, or both. The explanatory variables lesion area, perimeter and circularity were also square-root transformed to approximately follow a normal distribution. A detailed description of the modeling process is given elsewhere. ${ }^{50}$

The accuracy of all prognostic models was assessed in two clinically relevant scenarios using the coefficient of determination $\left(R^{2}\right)$ as an outcome measure. For scenario 1 'previously unknown patient' - leave-one-out cross-validation (LOO-CV) was carried out on the patient level (i.e., the model was fitted $n$ times to the data from $n-1$ patients using the remaining patient to validate the predictions). For prediction, the values of the patient-specific and eye-specific random effects were set to zero. For scenario 2 - 'prediction of the future progression rate with prior observation of the patient' - the visits from $n-1$ patients and all prior visits of the remaining patient (excluding the last visit) were used to fit the model, and the remaining last visit was used to test the predictions (LOO-CV on the visit level). For prediction, the estimated values of the patient-specific and eye-specific random effects were used. In a first step, the prognostic value of individual shape-descriptive factors was assessed. Further, a 'clinical trial inclusion model' including square root lesion area, FAF phenotype and focality index was probed, since these factors serve as eligibility criteria in currently ongoing clinical trials (NCT02247479, NCT02247531, NCT02087085) and last, a full model with all shape-descriptive factors. 


\section{Results}

Inter-rater reliability

Overall, the image grading and the subsequent automated measurement of the lesion area,

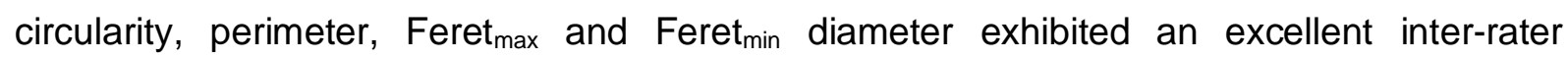
reliability (Figure 2). In a subset of 100 visits graded by two readers (M.L. and M.P.) blinded to the result of each other, the inter-rater reliability was $0.99(95 \% \mathrm{Cl} 0.99-0.99)$ for square root area, $0.99(0.98-0.99)$ for perimeter, 0.99 (0.98-0.99) for circularity, 0.97 (0.95-0.98) for Feret $_{\max }, 0.99(0.98-0.99)$ for Feret $_{\min }$ and $0.96(0.94-0.97)$ for focality index measurements. The Coefficients of Repeatability (CoR), i.e. the value below which the absolute differences between two ratings would lie within 0.95 probability, were $0.20 \mathrm{~mm}$ for square root area, $5.64 \mathrm{~mm}$ for perimeter, 0.07 for circularity, $1.03 \mathrm{~mm}$ for Feret ${ }_{\max }, 0.54$ for Feret $\min$ and 2.30 for focality index measurements (Figure 2). For all types of measurement, the Bland-Altmann plots showed no systematic differences (solid line) and narrow limits of agreement (dashed lines). A slightly increased inter-rater variability had been observed for measurements with higher values with the exception of area and circularity measurements (Figure 2).

\section{Cohort characteristics}

A total of 296 eyes of 201 patients (71 male [35.3\%], 130 female [64.7\%]) were included in this analysis. The mean age at baseline was 72.20 years \pm 13.08 . Most eyes presented with multifocal lesions (unifocal $42.2 \%$, oligofocal [2-4 foci] $34.5 \%$ and polyfocal [ $>4$ foci] $23.3 \%$ ). At baseline, the median [Q1; Q3] lesion area was $5.2 \mathrm{~mm}^{2}$ [2.3; 9.0] with a median perimeter and circularity of $16.5 \mathrm{~mm}[9.9 ; 26.4]$ and $0.2[0.1 ; 0.5]$, respectively (Table 1$)$. The mean square root progression rate was $0.31 \mathrm{~mm} /$ year. A detailed overview of the differences in baseline characteristics is given in Table 1 . Overall, there appeared to be an inverse association of the circularity with the previously published progression rates for the FAF phenotypes (Figure $3 \mathrm{~A}$ ) as confirmed by a correlation analysis (see Table, Supplemental 
Digital Content 1, which provides the pair-wise correlation analysis). All of the shapedescriptive factors were moderately (Pearson's $r>0.4)$ or strongly $(r>0.5)$ correlated to each other with few exceptions (see Figure, Supplemental Digital Content 2, showing the pair-wise correlation). As shown in Figure 4, the circularity differed markedly in dependence of the lesion configuration and was inversely correlated with the focality index.

\section{Prediction of GA progression based on individual shape-descriptive factors}

LOO-CV on the patient level (scenario 1: previously unknown patient - model without patient-specific intercepts and slopes) resulted in the following coefficients of determination $\left(R^{2}\right): 0.233$ for the Feret $\min$ model, 0.210 for the square-root circularity model, 0.098 for the square-root perimeter model, 0.088 for the FAF phenotype model, 0.056 for the focality index

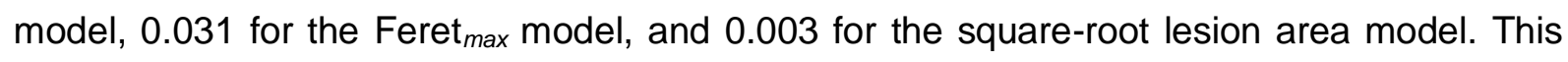

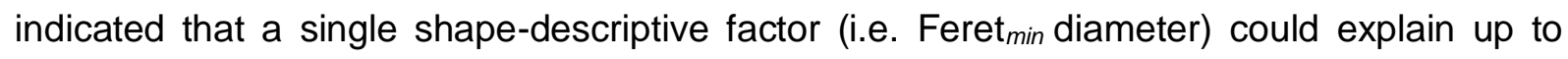
$23.3 \%$ of the variance in the progression rates in previously unknown patients (Table 2). Further, a model reflecting the current inclusion criteria for ongoing clinical trials (by including the variables square root lesion area, FAF phenotype and focality index) was evaluated for the first scenario. The coefficient of determination $\left(R^{2}=0.150\right)$ indicated that this model could explain $15 \%$ of the variance in the progression rates (Table 3 ). For the combined model, which included all covariates, the $R^{2}$ value was 0.244 indicating that these shape-descriptive factors (including the FAF phenotype) explained up to $24.4 \%$ of the variance in progression rates in previously unknown patients. To confirm that the results are not dependent on the disease severity, we performed the same analysis stratified according to lesion size (quartiles). Again, the circularity model exhibited the highest coefficient of determination among all shape-descriptive factors (see Table, Supplemental Digital Content 3, which shows the coefficient of determination for the stratified analysis).

For LOO-CV on the visit level (scenario 2: prediction of future progression rates with prior observation of the patient), the model without any shape-descriptive factors (autocorrelation 
of prior progression rates with future progression rates) resulted in a coefficient of determination $\left(R^{2}\right)$ of 0.185 indicating that previous progression rates explained $18.5 \%$ of the variance in future progression rates. In this second scenario, the models with the Feret min $_{\text {m }}$ diameter $\left(R^{2}=0.339\right)$, square-root circularity $\left(R^{2}=0.379\right)$, square-root perimeter $\left(R^{2}=0.344\right)$, FAF phenotype $\left(R^{2}=0.325\right)$, focality index $\left(R^{2}=0.335\right)$, Feret $\max$ diameter $\left(R^{2}=0.330\right)$ and square-root lesion area $\left(R^{2}=0.354\right)$ were more accurate in predicting future progression rates. For the combined model with all covariates, the cross-validated $R^{2}$ value was 0.391 in the scenario 2 indicating that the evaluated shape-descriptive factors (including the FAF phenotype) explained up to $39.1 \%$ of the variance in progression rates with prior observation of the patient. Since diffuse-trickling GA exhibits a different genetic risk profile compared to other GA phenotypes (cf. Discussion) ${ }^{51}$, the aforementioned analyses were also carried out

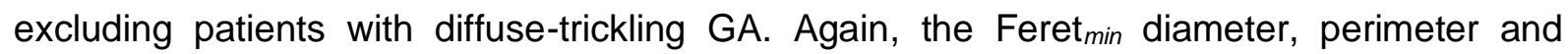
circularity revealed the highest prognostic value for future progression rates (see Table Supplemental Digital Content 4, which shows the results for the analysis excluding patients with diffuse-trickling $G A$ ).

\section{Phenotypic differences in GA progression}

Subgroup analysis of the FAF phenotype with the lowest circularity (i.e. diffuse-trickling GA) revealed that these patients $(n=25[12.4 \%])$ were younger than patients with non-diffusetrickling GA (69.4 years \pm 8.3 versus 74.7 years \pm 6.9$)$. This difference was statistically significant $(p=0.0085)$.

The median baseline perimeter was larger in diffuse-trickling GA versus non-diffuse-trickling GA with values of 32.3 [25.5; 40.3] versus 15.0 [9.0;23.4] mm, respectively. Thus, despite of the larger baseline lesion size $(9.8[5.2 ; 13.6]$ vs. 4.8 [2.1; 8.4]), diffuse-trickling GA exhibited a much lower circularity than non-diffuse-trickling GA $(0.1[0.1 ; 0.2]$ vs. $0.3[0.1 ; 0.5])$. Likewise, the Feret $_{\max }$ and Feret min $_{\text {diameters }}$ were larger in diffuse-trickling GA as compared to non-diffuse-trickling GA (Feret $\max _{\max } 1545.0$ [1224.0; 1932.0] vs. 1115.0 [793.3; 1351.1] and 
Feret $_{\min } 1076.3[916.7 ; 1274.7]$ vs. $\left.860.6[571.9 ; 1067.1]\right)$. A detailed overview is shown in Table 3 and Figure 3B.

Exploratory analysis of individual eyes disclosed that non-diffuse-trickling GA showed progression mainly through 'appositional progression'. Typically, areas of GA exhibited progression in continuity with the former lesion boundary (Figure 5). In contrast, progression in diffuse-trickling GA appeared to be driven through both, 'appositional progression' and formation of 'de-novo' foci and lobules. These 'de-novo' foci were typically in close proximity to the former lesion boundary, but not in direct continuity with the former boundary. This appeared to result in increased perimeter values and low circularity values. Figure 5 shows six exemplary eyes of six patients ( 3 with non-diffuse-trickling GA and 3 with diffuse-trickling GA) with a follow-up of 12 or 18 months each. 


\section{Discussion}

The findings underscore the prognostic relevance of shape-descriptive factors on the progression of GA. In particular, the parameters circularity, perimeter, focality index and

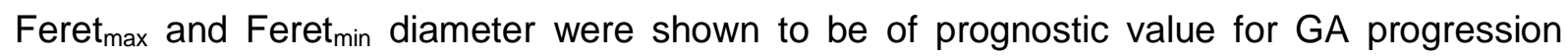
explaining individually up to $23.3 \%$ (e.g. Feret min $_{\text {in }}$ previously unknown patients) and $37.9 \%$ (e.g. circularity with prior observation of the patients) of the variance in progression rates. Further, circularity appeared to be inversely associated with the previously published progression rates for the FAF phenotypes. ${ }^{25}$ The combined mixed-effects model with lesion

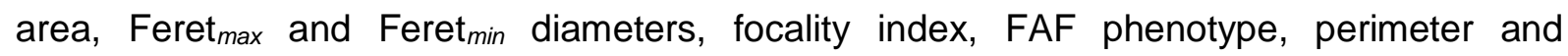
circularity as prognostic variables explained (cross-validated) $39.1 \%$ of the variability in progression rates with prior observation of the patient.

The model based on square-root lesion area exhibited a very low $R^{2}$ value, while the model based on the square-root circularity exhibited a high $R^{2}$ value. These findings are in accordance with previous reports by Yehoshua and coworkers, who demonstrated that square-root transformation of lesion area measurements (based on SD-OCT) reduces the association between progression rates and baseline area. ${ }^{29,38}$ This notion of quasiexponential progression kinetics (i.e. progression of GA being proportional to the lesion circumference) has also been previously reported and is based on circularity measurements using CFPs. ${ }^{36}$ With regard to the underlying pathogenic mechanisms, it is suggested that a cascading process at the lesion boundary drives GA progression . In a recent histopathologic study, Zanzottera and coworkers were able to confirm quantitatively that the percentage of abnormal RPE cells with 'sloughed', 'shedding', and 'intraretinal' morphologies increases towards the boundary of atrophy (approached from the non-atrophic side),- - an observation that had been previously described by Sarks et al. in $1988 .{ }^{13,14}$ Further, the authors noted that focally increased FAF at the junctional zone of GA most likely represents RPE cell dysmorphia and displacement including rounding and anterior migration rather than intracellular lipofuscin accumulation. ${ }^{14,52-54}$ In conjunction with the here reported findings this 
could imply that the GA perimeter and FAF phenotype directly reflect the degree of severely abnormal RPE cells in the border zone of eyes with GA and are thus associated with the progression rates. The Feret diameters - also prognostic for the progression rates - appear to also reflect the planar extent of diseased RPE cells. Notably, all of these factors were also prognostic in the analysis excluding diffuse-trickling GA. In diffuse-trickling geographic atrophy, GA progression appears to be mediated predominantly by 'de-novo' foci formation and not 'appositional progression'. Thus, there might be differences in the underlying pathogenesis when compared with non-diffuse-trickling GA that are also reflected by the much lower circularity values for diffuse-trickling GA. Since the lobules in diffuse-trickling GA typically manifest a diameter of $600-1000 \mu \mathrm{m}$ (similar size to choriocapillaris lobules in humans [range of 420 to $1200 \mu \mathrm{m}$ ]), ${ }^{55}$ choroidal insufficiency in association with basal laminar deposits has been previously implied to possibly contribute to the pathogenesis of diffuse-trickling GA. ${ }^{56,57}$ Delayed or reduced filling of the choriocapillaris in fluorescein angiography, pronounced choroidal thinning in SD-OCT, and the higher prevalence of cardiovascular comorbidity further support the hypothesis that choroidal alterations may be involved in this distinct GA phenotype. ${ }^{56,58}$

Understanding GA progression and its individual variability is also important for the design and interpretation of interventional clinical trials as well as for patient education and counseling. ${ }^{9}$ However, a substantial part of the remaining variation in GA progression appears to depend on additional variables, which remain to be elucidated. While lesion size,${ }^{23-31}$ previous lesion progression, ${ }^{32}$ lesion shape, ${ }^{23,26,33-38}$ and focality index, ${ }^{26,31,33-38}$ have been addressed in this study, other variables, including lesion topography ${ }^{13,21,23,26,32,39}$ or SD-OCT characteristics ${ }^{37,59-62}$ have not been addressed. Thus, incorporating additional information from other imaging modalities, especially SD-OCT, which was recently recommended by the CAM (Classification of Atrophy Meetings) group as the reference method for defining different atrophy phenotypes, ${ }^{62}$ may increase the prognostic accuracy. ${ }^{60,63}$ Interestingly, a study by Niu et al. on 29 patients evaluating quantitative SD- 
OCT biomarkers reported a correlation coefficient of 0.74 for the predicted progression rate of previously unknown patients (comparable to scenario 1 in this study) and a correlation coefficient 0.72 for patients with previous observation records (comparable to scenario 2 in this study), translating to a $R^{2}$ values of 0.55 and 0.52 , respectively ${ }^{64}$ Compared to the here reported $\mathrm{R}^{2}$ value of 0.39 for scenario 2 , the results obtained by Niu et al. suggest that SDOCT might indeed add prognostic information over and above FAF imaging and CFP. Importantly, the most predictive SD-OCT feature reported by Niu et al. is confined to the outer retina and RPE (bands 11 to 14 ) that would also include reticular drusen, drusen and basal laminar deposits. ${ }^{60}$ The FAF signal is thought to derive from alterations located in these retinal layers. ${ }^{65}$ Therefore, the FAF phenotype may reflect to some extent these SD-OCT features, highlighting that identification of independent (uncorrelated) prognostic factors will be needed to refine the prognostic accuracy of the models.

Genetic, epigenetic, environmental and demographic factors may also be of prognostic relevance for GA enlargement rates. ${ }^{9}$ While these factors were not evaluated in this study, their maximal possible effect sizes may be deduced (with caution) and estimated. To date, none of the examined single nucleotide polymorphism (SNP) could consistently be linked to GA progression. ${ }^{66-69}$ However, by combining the FAM- and AREDS-study patient cohorts ARMS2_rs10490924, C3_rs2230199 and presence of GA in the fellow eye were reported to significantly contribute to GA progression, thus jointly accounting for up to $7.2 \%$ of the variability in progression rates. ${ }^{66}$ In accordance with findings obtained by Sunness et al., our data showed that the autocorrelation between previous progression rates and future progression rates could account for approximately $20 \%$ of the variance in progression rates $^{32}$ It is likely that the effects of genotype, environment and lifestyle remained stable in most patients throughout the course of this study and are therefore already represented by the autocorrelation between previous and future progression rates. 
Several limitations of this study need to be considered. First, the quantification method for perimeter, circularity and Feret diameters is novel. While the quantification of the GA area based on FAF images using the RegionFinder software is predominantly independent of the FAF image quality, perimeter measurements depend on the smoothness of the GA boundaries (and thus on the FAF image quality). To circumvent this limitation, all annotations were equally smoothed prior to quantification (cf., Figure 1). For these measurements, reading center standard operating procedures will become necessary to define limits of agreement. Second, the here described models predict the progression of complete RPE and outer retinal atrophy (cRORA) as determined by FAF. ${ }^{62}$ However, atrophy of the outer retina in absence of RPE atrophy has been described in eyes with non-exudative AMD, especially in eyes with reticular drusen. ${ }^{62,70,71}$ While these types of lesions were not assessed in this study and could therefore not be modeled as outcome variable, it must be considered that the extent of lesions characterized by incomplete RPE atrophy appears to be correlated to the extent of cRORA. ${ }^{70}$ Thrid SD-OCT data, genetic, epigenetic, environmental and demographic factors were not considered as explanatory variables in this study. While variegated lesion with involvement of the RPE may be detected using FAF imaging, ${ }^{65}$ lesions strictly confined to the neurosensory retina such as "outer retinal tabulations", which could be indicative of Müller cell remodeling, may only be resolved using SD-OCT imaging. ${ }^{72,73} \mathrm{~A}$ direct comparison of all of these markers in the same cohort appears mandatory for a comparative analysis of their respective effect sizes on GA progression. Finally, machine learning approaches might allow for an even more precise prediction of progression rates from FAF images. While limited, this study had a relatively large sample size of 296 eyes of 201 patients with a median follow-up [Q1-Q3] of 2.38 years [1.46-4.24].

The findings herein confirm the importance of lesion shape characteristics as prognostic variables for GA progression in addition to baseline lesion size, lesion location, focality index and fellow eye status. Since evaluation of these lesion shape-descriptive factors could be performed fully automatically, based on FAF annotations that are already part of the eligibility assessment of currently ongoing trials, it would be feasible to include these factors as 
selection criteria for future clinical studies and interventional trials. As shown in this study, these factors would allow for a more accurate prediction of progression rates over time as compared to currently used selection criteria. 


\section{References}

1. Klein R, Klein BEK, Knudtson MD, Meuer SM, Swift M, Gangnon RE. Fifteen-year cumulative incidence of age-related macular degeneration: the Beaver Dam Eye Study. Ophthalmology 2007; 114:253-262.

2. Lim LS, Mitchell P, Seddon JM, Holz FG, Wong TY. Age-related macular degeneration. Lancet 2012; 379:1728-1738.

3. Freund KB, Ho I-V, Barbazetto IA, et al. Type 3 neovascularization: the expanded spectrum of retinal angiomatous proliferation. Retina 2008; 28:201-211.

4. Li M, Dolz-Marco R, Messinger JD, et al. Clinicopathologic Correlation of Anti-Vascular Endothelial Growth Factor-Treated Type 3 Neovascularization in Age-Related Macular Degeneration. Ophthalmology September 2017.

5. Gass JD. Drusen and disciform macular detachment and degeneration. Trans Am Ophthalmol Soc 1972; 70:409-436.

6. Blair CJ. Geographic atrophy of the retinal pigment epithelium: A manifestation of senile macular degeneration. Arch Ophthalmol 1975; 93:19-25.

7. Schatz H, McDonald HR. Atrophic macular degeneration. Rate of spread of geographic atrophy and visual loss. Ophthalmology 1989; 96:1541-1551.

8. Sunness JS. The natural history of geographic atrophy, the advanced atrophic form of age-related macular degeneration. Mol Vis 1999; 5:25-30.

9. Cheung LK, Eaton A. Age-Related Macular Degeneration. Pharmacother J Hum Pharmacol Drug Ther 2013; 33:838-855.

10. Schmitz-Valckenberg S, Nadal J, Fimmers R, et al. Modeling Visual Acuity in Geographic Atrophy Secondary to Age-Related Macular Degeneration. Ophthalmologica 2016; 235:215-224. 
11. Schmitz-Valckenberg S, Sadda S, Staurenghi G, et al. Geographic Atrophy. Retina 2016; 36:2250-2264.

12. Schmitz-Valckenberg S. The Journey of "Geographic Atrophy" through Past, Present, and Future. Ophthalmologica January 2017.

13. Sarks JP, Sarks SH, Killingsworth MC. Evolution of geographic atrophy of the retinal pigment epithelium. Eye (Lond) 1988; 2:552-577.

14. Zanzottera EC, Ach T, Huisingh C, Messinger JD, Freund KB, Curcio CA. Visualizing Retinal Pigment Epithelium Phenotypes in the Transition To Atrophy in Neovascular Age-Related Macular Degeneration. Retina 2016; 36:S26-S39.

15. Lambert NG, EIShelmani H, Singh MK, et al. Risk factors and biomarkers of agerelated macular degeneration. Prog Retin Eye Res 2016; 54:64-102.

16. Delori FC, Dorey CK, Staurenghi G, Arend O, Goger DG, Weiter JJ. In vivo fluorescence of the ocular fundus exhibits retinal pigment epithelium lipofuscin characteristics. Invest Ophthalmol Vis Sci 1995; 36:718-729.

17. von Rückmann a, Fitzke FW, Bird a C, von Ruckmann A, Fitzke FW, Bird a C. Distribution of fundus autofluorescence with a scanning laser ophthalmoscope. $\mathrm{Br} \mathrm{J}$ Ophthalmol 1995; 79:407-412.

18. Bindewald A, Bird AC, Dandekar SSS, et al. Classification of fundus autofluorescence patterns in early age-related macular disease. Invest Ophthalmol Vis Sci 2005; 46:3309-3314.

19. Deckert A, Schmitz-Valckenberg S, Jorzik J, Bindewald A, Holz FG, Mansmann U. Automated analysis of digital fundus autofluorescence images of geographic atrophy in advanced age-related macular degeneration using confocal scanning laser ophthalmoscopy (cSLO). BMC Ophthalmol 2005; 5:8.

20. Schmitz-Valckenberg S, Brinkmann CK, Alten F, et al. Semiautomated image 
processing method for identification and quantification of geographic atrophy in agerelated macular degeneration. Invest Ophthalmol Vis Sci 2011; 52:7640-7646.

21. Lindner M, Böker A, Mauschitz MM, et al. Directional Kinetics of Geographic Atrophy Progression in Age-Related Macular Degeneration with Foveal Sparing.

Ophthalmology 2015; 122:1356-1365.

22. Sadda SR, Chakravarthy U, Birch DG, Staurenghi G, Henry EC, Brittain C. Clinical Endpoints for the Study of Geographic Atrophy Secondary To Age-Related Macular Degeneration. Retina 2016; 36:1806-1822.

23. Sunness JS, Gonzalez-Baron J, Applegate CA, et al. Enlargement of atrophy and visual acuity loss in the geographic atrophy form of age-related macular degeneration. Ophthalmology 1999; 106:1768-1779.

24. Lindblad AS, Lloyd PC, Clemons TE. Change in Area of Geographic Atrophy in the Age-Related Eye Disease Study. Arch Ophthalmol 2009; 127:1168-1174.

25. Holz FG, Bindewald-Wittich A, Fleckenstein M, Dreyhaupt J, Scholl HPN, SchmitzValckenberg S. Progression of Geographic Atrophy and Impact of Fundus Autofluorescence Patterns in Age-related Macular Degeneration. Am J Ophthalmol $2007 ; 143: 463-472$

26. Schmitz-Valckenberg S, Sahel JA, Danis R, et al. Natural History of Geographic Atrophy Progression Secondary to Age-Related Macular Degeneration (Geographic Atrophy Progression Study). Ophthalmology 2016; 123:361-368.

27. Biarnés M, Arias L, Alonso J, et al. Increased Fundus Autofluorescence and Progression of Geographic Atrophy Secondary to Age-Related Macular Degeneration: The GAIN Study. Am J Ophthalmol 2015; 160:345-353.e5.

28. Batioglu F, Oguz YG, Demirel S, Özmert E. Geographic atrophy progression in eyes with age-related macular degeneration: Role of fundus autofluorescence patterns, 
fellow eye and baseline atrophy area. Ophthalmic Res 2014; 52:53-59.

29. Feuer WJ, Yehoshua Z, Gregori G, et al. Square Root Transformation of Geographic Atrophy Area Measurements to Eliminate Dependence of Growth Rates on Baseline Lesion Measurements: A Reanalysis of Age-Related Eye Disease Study Report No. 26. JAMA Ophthalmol 2013; 131:110-111.

30. Jeong YJ, Hong IH, Chung JK, Kim KL, Kim HK, Park SP. Predictors for the progression of geographic atrophy in patients with age-related macular degeneration: fundus autofluorescence study with modified fundus camera. Eye (Lond) 2014; 28:209-218.

31. Marsiglia M, Boddu S, Bearelly S, et al. Association between geographic atrophy progression and reticular pseudodrusen in eyes with dry age-related macular degeneration. Invest Ophthalmol Vis Sci 2013; 54:7362-7369.

32. Sunness JS, Margalit E, Srikumaran D, et al. The Long-term Natural History of Geographic Atrophy from Age-Related Macular Degeneration. Enlargement of Atrophy and Implications for Interventional Clinical Trials. Ophthalmology 2007; 114:271-277.

33. Klein R, Meuer SM, Knudtson MD, Klein BEK. The Epidemiology of Progression of Pure Geographic Atrophy: The Beaver Dam Eye Study. Am J Ophthalmol 2008; 146:692-699.

34. Joachim N, Mitchell P, Kifley A, Rochtchina E, Hong T, Wang JJ. Incidence and progression of geographic atrophy: Observations from a population-based cohort. Ophthalmology 2013; 120:2042-2050.

35. Xu L, Blonska AM, Pumariega NM, et al. Reticular Macular Disease Is Associated With Multilobular Geographic Atrophy in Age-Related Macular Degeneration. Retina 2013; 33:1850-1862.

36. Domalpally A, Danis RP, White J, et al. Circularity index as a risk factor for 
progression of geographic atrophy. Ophthalmology 2013; 120:2666-2671.

37. Moussa K, Lee JY, Stinnett SS, Jaffe GJ. Spectral Domain Optical Coherence Tomography-Determined Morphologic Predictors of Age-Related Macular Degeneration-Associated Geographic Atrophy Progression. Retina 2013; 33:15901599.

38. Yehoshua Z, Rosenfeld PJ, Gregori G, et al. Progression of geographic atrophy in age-related macular degeneration imaged with spectral domain optical coherence tomography. Ophthalmology 2011; 118:679-686.

39. Mauschitz MM, Fonseca S, Chang P, et al. Topography of geographic atrophy in agerelated macular degeneration. Invest Ophthalmol Vis Sci 2012; 53:4932-4939.

40. Schmitz-Valckenberg S, Bindewald-Wittich A, Dolar-Szczasny J, et al. Correlation between the area of increased autofluorescence surrounding geographic atrophy and disease progression in patients with AMD. Invest Ophthalmol Vis Sci 2006; 47:26482654.

41. Mones J, Biarnes M. Geographic atrophy phenotype identification by cluster analysis. Br J Ophthalmol July 2017.

42. Ferris FL, Wilkinson CP, Bird A, et al. Clinical Classification of Age-related Macular Degeneration. Ophthalmology 2013; 120:844-851.

43. Fritsche LG, Fleckenstein M, Fiebig BS, et al. A Subgroup of Age-Related Macular Degeneration is Associated With Mono-Allelic Sequence Variants in the ABCA4 Gene. Invest Ophthalmol Vis Sci 2012; 53:2112.

44. Smailhodzic D, Fleckenstein M, Theelen T, et al. Central areolar choroidal dystrophy $(C A C D)$ and age-related macular degeneration (AMD): differentiating characteristics in multimodal imaging. Invest Ophthalmol Vis Sci 2011; 52:8908-8918.

45. Pfau M, Goerdt L, Schmitz-Valckenberg S, et al. Green-Light Autofluorescence Versus 
Combined Blue-Light Autofluorescence and Near-Infrared Reflectance Imaging in Geographic Atrophy Secondary to Age-Related Macular Degeneration. Invest Ophthalmol Vis Sci 2017; 58:BIO121-BIO130.

46. Schneider CA, Rasband WS, Eliceiri KW. NIH Image to ImageJ: 25 years of image analysis. Nat Methods 2012; 9:671-675.

47. Feret LR. La grosseur des grains. Assoc Intern Essais Math D 1931; 2 .

48. R Development Core Team. R: A Language and Environment for Statistical Computing. 2012.

49. Bland JM, Altman DG. Statistical methods for assessing agreement between two methods of clinical measurement. Lancet 1986; 1:307-310.

50. Dreyhaupt J, Mansmann U, Pritsch M, Dolar-Szczasny J, Bindewald a, Holz FG. Modelling the natural history of geographic atrophy in patients with age-related macular degeneration. Ophthalmic Epidemiol 2005; 12:353-362.

51. Fleckenstein M, Grassmann F, Lindner M, et al. Distinct genetic risk profile of the rapidly progressing diffuse-trickling subtype of geographic atrophy in age-related macular degeneration (AMD). Invest Ophthalmol Vis Sci 2016; 57:2463-2471.

52. Zanzottera EC, Messinger JD, Ach T, Theodore Smith R, Curcio CA. Subducted and melanotic cells in advanced age-related macular degeneration are derived from retinal pigment epithelium. Invest Ophthalmol Vis Sci 2015; 56:3269-3278.

53. Zanzottera EC, Messinger JD, Ach T, Theodore Smith R, Bailey Freund K, Curcio CA. The project macula retinal pigment epithelium grading system for histology and optical coherence tomography in age-related macular degeneration. Invest Ophthalmol Vis Sci 2015; 56:3253-3268.

54. Ach T, Tolstik E, Messinger JD, Zarubina A V., Heintzmann R, Curcio CA. Lipofuscin redistribution and loss accompanied by cytoskeletal stress in retinal pigment 
epithelium of eyes with age-related macular degeneration. Invest Ophthalmol Vis Sci $2015 ; 56: 3242-3252$.

55. Torczynski E, Tso MO. The architecture of the choriocapillaris at the posterior pole. Am J Ophthalmol 1976; 81:428-440.

56. Fleckenstein M, Schmitz-Valckenberg S, Lindner M, et al. The "Diffuse-Trickling" Fundus autofluorescence phenotype in geographic atrophy. Invest Ophthalmol Vis Sci $2014 ; 55: 2911-2920$.

57. McLeod DS, Lutty GA. High-resolution histologic analysis of the human choroidal vasculature. Invest Ophthalmol Vis Sci 1994; 35:3799-3811.

58. Lindner M, Bezatis A, Czauderna J, et al. Choroidal Thickness in Geographic Atrophy Secondary to Age-Related Macular Degeneration. Invest Ophthalmol Vis Sci 2015; 56:875-882.

59. Fleckenstein M, Schmitz-Valckenberg S, Martens C, et al. Fundus autofluorescence and spectral-domain optical coherence tomography characteristics in a rapidly progressing form of geographic atrophy. Invest Ophthalmol Vis Sci 2011; 52:37613766.

60. Niu S, de Sisternes L, Chen Q, Rubin DL, Leng T. Fully Automated Prediction of Geographic Atrophy Growth Using Quantitative Spectral-Domain Optical Coherence Tomography Biomarkers. Ophthalmology 2016; 123:1737-1750.

61. Holz FG, Sadda SR, Staurenghi G, et al. Imaging Protocols in Clinical Studies in Advanced Age-Related Macular Degeneration: Recommendations from Classification of Atrophy Consensus Meetings. Ophthalmology 2017; 124:464-478.

62. Sadda SR, Guymer R, Holz FG, et al. Consensus Definition for Atrophy Associated with Age-Related Macular Degeneration on OCT: Classification of Atrophy Report 3. Ophthalmology November 2017. 
63. Dysli C, Wolf S, Zinkernagel MS. Autofluorescence Lifetimes in Geographic Atrophy in Patients With Age-Related Macular Degeneration. Invest Ophthalmol Vis Sci 2016; 57:2479.

64. Niu S, de Sisternes L, Chen Q, Leng T, Rubin DL. Automated geographic atrophy segmentation for SD-OCT images using region-based C-V model via local similarity factor. Biomed Opt Express 2016; 7:581.

65. Oishi M, Oishi A, Lindner M, et al. Structural Changes in Optical Coherence Tomography Underlying Spots of Increased Autofluorescence in the Perilesional Zone of Geographic Atrophy. Invest Ophthalmol Vis Sci 2017; 58:3303-3310.

66. Grassmann F, Fleckenstein M, Chew EY, et al. Clinical and genetic factors associated with progression of geographic atrophy lesions in age-related macular degeneration. PLoS One 2015; 10:e0126636.

67. Scholl HPN, Fleckenstein M, Fritsche LG, et al. CFH, C3 and ARMS2 are significant risk loci for susceptibility but not for disease progression of geographic atrophy due to AMD. PLoS One 2009; 4:e7418.

68. Klein ML, lii FLF, Francis PJ, et al. Progression of Geographic Atrophy and Genotype in Age- Related Macular Degeneration. Ophthalmology 2011; 117:1554-1559.

69. Caire J, Recalde S, Velazquez-Villoria A, et al. Growth of geographic atrophy on fundus autofluorescence and polymorphisms of CFH, CFB, C3, FHR1-3, and ARMS2 in age-related macular degeneration. JAMA Ophthalmol 2014; 132:528-534.

70. Sayegh RG, Simader C, Scheschy U, et al. A systematic comparison of spectraldomain optical coherence tomography and fundus autofluorescence in patients with geographic atrophy. Ophthalmology 2011; 118:1844-1851.

71. Spaide RF. Outer Retinal Atrophy After Regression of Subretinal Drusenoid Deposits As a Newly Recognized Form of Late Age-Related Macular Degeneration. Retina 
2013; 33:1800-1808.

72. Dolz-Marco R, Litts KM, Tan ACS, Freund KB, Curcio CA. The Evolution of Outer Retinal Tubulation, a Neurodegeneration and Gliosis Prominent in Macular Diseases. Ophthalmology 2017; 124:1353-1367.

73. Edwards MM, McLeod DS, Bhutto IA, Grebe R, Duffy M, Lutty GA. Subretinal Glial Membranes in Eyes With Geographic Atrophy. Invest Ophthalmol Vis Sci 2017; $58: 1352-1367$. 


\section{Figure legends}

\section{Figure 1}

Delineation of areas of definitely decreased autofluorescence was performed using a semiautomated image analyses software (RegionFinder, Heidelberg Engineering). The version (2.5.5.0) includes a feature that automatically registers FAF $(\mathbf{A})$ to corresponding NIR (B) reflectance images. The graded annotated images (C) were transferred to ImageJ (Bethesda, Maryland, USA) to measure the area (C), perimeter (D), Feret max $_{\text {(E) }}$ and Feret min $_{\text {in }}$ diameter $(\mathbf{F})$ of the lesions with a costume ImageJ plug-in. The circularity was calculated as the ratio of $4 \times \pi \times$ area to the perimeter squared. The circularity of a perfectly circular lesion would be "1.0", while more irregular shaped lesions would exhibit lower values for circularity.

\section{Figure 2}

Bland Altman plots for the inter-rater reliability of the square root (sqrt) area (A), perimeter

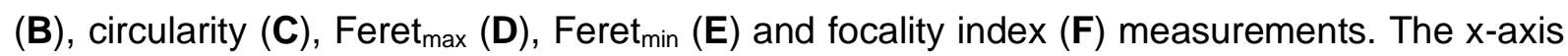
shows the mean value of each measurement. The difference between two measurements (rater $1-$ rater 2 ) is indicated on the y-axis. The overall mean difference is illustrated by the solid line; the $95 \%$ limits of agreement are marked by the two dashed lines. In the plot for the focality index measurements (F), the size of individual circles illustrates the count of overlapping data points. 


\section{Figure 3. Circularity in dependence of the FAF phenotype}

Panel A shows the circularity for each of the FAF phenotypes. Note that the circularity appeared to be inversely associated with the previously published progression rates for the FAF phenotypes. ${ }^{25}$ Hereby, diffuse-trickling GA exhibited the lowest circularity associated with the highest progression rates. Panel B depicts the circularity in dependence of the simplified FAF phenotype classification (diffuse-trickling GA versus non-diffuse-trickling GA).

\section{Figure 4. Circularity for various exemplary GA lesions}

The figure shows the FAF images of six eyes with GA. The number in the lower left corner of each FAF image indicates the circularity of the lesion. Please note that lesions with a low circularity tend to be polyfocal ( $>4$ foci) whereas lesions with a higher circularity tend to be oligofocal (>1 - $\leq 4$ foci) or unifocal. Through this, unifocal lesions with a high circularity (that tend to progress slowly) may be identified in a clinical setting.

\section{Figure 5. Exemplary cases}

The eyes with non-diffuse-trickling GA (first and second column) exhibited overall a high circularity and slow GA progression during the follow-up period ( $\mathrm{M} 0$ - month $0, \mathrm{M} 12$ - month 12). The third eye with non-diffuse-trickling GA (first and second column, third row) showed a relatively lower circularity as compared to the other two eyes with non-diffuse-trickling GA and a more rapid progression rate. Eyes with diffuse-trickling GA (third and forth column) exhibited overall a low circularity associated with rapid progression rates. Note, while the eyes with non-diffuse-trickling GA showed primarily progression through 'appositional expansion', the eyes with diffuse-trickling GA exhibited progression through upcoming 'denovo' foci and lobules. Further, the FAF signal within areas of atrophy was brighter ('grayish') in diffuse-trickling GA as compared to non-diffuse-trickling GA. 


\section{List of Supplemental Digital Content}

Supplemental Digital Content 1. Table that provides the pair-wise correlation analysis. pdf Supplemental Digital Content 2. Figure that shows the pair-wise correlation. tiff Supplemental Digital Content 3. Table that shows the coefficient of determination for the stratified analysis. pdf

Supplemental Digital Content 4. Table that shows the results for the analysis excluding patients with diffuse-trickling GA. pdf 